\title{
Retrospective Cohort Study Showing Clinical Equivalence of Microendoscopic Laminotomy to Open Fenestration for Patients with Lumbar Spinal Stenosis
}

\author{
Takahito Fujimori ${ }^{1}$, Yoshihiro Mukai ${ }^{2}$, Nozomu Nakajima ${ }^{1}$, Tsuyoshi Sugiura ${ }^{1}$, Daisuke Ikegami ${ }^{1}$, Hironobu Sakaura ${ }^{1}$ \\ ${ }^{1}$ Department of Orthopedic Surgery, Japan Community Healthcare Organization Osaka Hospital, Osaka, Japan \\ ${ }^{2}$ Department of Orthopedic Surgery, Nishinokyo Hospital, Nara, Japan
}

Corresponding Author:

Takahito Fujimori, MD

Department of Orthopedic Surgery, Japan Community Healthcare

Organization, Osaka Hospital, 4-2-78, Fukushimaku, Osaka, Japan, 553-0003

Tel: +81-6-6441-5451

Fax: +81-6-6445-8900

E-mail: takahito-f@hotmail.co.jp

Received: September 14, 2020

Revised: October 29, 2020

Accepted: November 09, 2020

\begin{abstract}
Objective: Despite the popularity of microendoscopic disectomy, there is currently insufficient studies about microendoscopic laminotomy (MEL) for lumbar spinal stenosis. The purpose of this study was to compare the clinical and radiographic outcomes of MEL and fenestration (laminotomy in open procedure) for lumbar spinal stenosis. Methods: This study included 30 patients in the MEL group and 46 patients in the open fenestration group between 2012 and 2016 (follow-up period $\geq 1$ year). The Japanese Orthopedic Association Back Pain Evaluation Questionnaire (JOABPEQ), a visual analog scale (VAS), surgical outcomes, blood test outcomes, and radiographic parameters were studied. Results: Mean age was 67 years old in the MEL group and 70 years old in the open fenestration group $(p=0.1)$. There were no significant differences in score change of either domain of JOABPEO between MEL and fenestration. The 95\% confidence intervals of the between-group differences in score change were within clinical important difference ( \pm 20 point) in all the domains of JOABPEO. The MEL group had significantly shorter hospital stays ( 9 days vs 13 days; $p<0.001$ ), smaller increase in C-reactive protein $(1.7 \mathrm{mg} / \mathrm{dL}$ vs $2.9 \mathrm{mg} / \mathrm{dL} ; \mathrm{p}=0.009)$, and longer operating time (122 $\mathrm{min}$ vs $39 \mathrm{~min} ; \mathrm{p}<$ $0.001)$ than the fenestration group. There was no significant difference in hemoglobin level, total protein, albumin, creatine kinase between the groups. The MEL group had one case of dural tear and the fenestration group had two cases $(p=1.0)$. There was no significant differences in complication rate between the groups. There were no significant between-group differences in change of disc height or ROM. Conclusion: In the treatment of lumbar spinal stenosis, the clinical effectiveness and safety of MEL was equivalent to that of fenestration, with less invasiveness.
\end{abstract}

Key Words: Microendoscopic laminotomy, Fenestration, Minimally invasive, Decompression, Facet resection, Outcome

\section{INTRODUCTION}

Along with advances in technology, microendoscopic spinal surgery using the METRx system has become popular'). Currently, the indication of microendoscopic spinal surgery is extended not only to simple lumbar herniation, but also to more degenerative lumbar spinal stenosis (microendoscopic laminotomy: MEL) ${ }^{2,3)}$. According to reports from the endoscopic surgical skill qualification system of the Japanese Orthopedic Association, approximately 15,000 cases of microendoscopic spinal surgery are perfor- med annually in Japan ${ }^{2)}$.

Despite the popularity of microendoscopic spinal surgery, there is currently insufficient evidence to support its effectiveness, and less invasive nature ${ }^{4)}$. In open procedures, surgeons have the luxury of wide exposure to enable identification of anatomy and multiple trajectories for tissue manipulation. On the other hand, in microendoscopic spinal surgery, surgeons need to manipulate operative instruments one-handed with restrictive trajectories due to a tubular retractor in a small, non-stereoscopic, and obliquely-viewed surgical field. This technical difficulty might disorient surgeons, resulting in insufficient decom- 
pression of nerves, dural tear, or iatrogenic pars fracture due to over-resection of the facet ${ }^{4,5)}$.

Currently, there are few studies comparing MEL and open laminotomy. The purpose of this study was to compare the clinical and radiographic outcomes of MEL with those of conventional open procedures.

\section{MATERIALS AND METHODS}

\section{Study Participants}

Our study protocol was approved by our institutional review board. This was a retrospective study in a single spine center between March 2012 and December 2016. Inclusion criteria for the study were: (1) leg pain or neurogenic claudication due to radiculopathy and/or cauda equina syndrome with or without low back pain, (2) diagnosis of lumbar canal stenosis refractory to conservative management, (3) planned L4/5 microendoscopic laminotomy, (4) pre- and post-operative outcome, (5) a minimum follow-up period of 1 year. Patients with history of past spinal surgery, rheumatoid arthritis, hemodialysis for chronic renal failure were excluded from this study.

The control group included age and sex-matched patients who underwent conventional fenestration (laminotomy in open procedure) at $L 4 / 5$ during the same period. In this spine center, five attending surgeons, who were approved by the Board of the Japanese Society for Spine Surgery and Related Research, performed the surgeries. Two of the five surgeons were certified by the Japanese Orthopedic Association with an endoscopic surgical skill qualification. These two surgeons performed the MEL procedures. The other three surgeons performed only conventional open fenestration.

The demographic data of the MEL and fenestration groups are shown in Table 1.

\section{Surgical Technique}

\section{1) Microendoscopic Laminotomy (MEL)}

The MEL method has been reported in the previous literature ${ }^{6)}$. Our L4/5 MEL procedures were as follows (see Figure 1). A longi-

Table 1. Comparison of demographic data between the MEL group and the fenestration group

\begin{tabular}{|c|c|c|c|}
\hline Group & MEL & Fenestration & $p$-value \\
\hline N & 30 & 46 & N.A. \\
\hline Female & 8 & 13 & 0.7 \\
\hline Age & $67 \pm 7$ & $70 \pm 10$ & 0.1 \\
\hline Body mass index $\left(\mathrm{kg} / \mathrm{m}^{2}\right)$ & $24.5 \pm 3.2$ & $24.3 \pm 3.4$ & 0.9 \\
\hline Follow-up period (day) & $377 \pm 38$ & $371 \pm 30$ & 0.4 \\
\hline Charlson comorbidity index & $0.6 \pm 0.9$ & $0.9 \pm 1.0$ & 0.2 \\
\hline Decompression Unilateral & 3 & 0 & 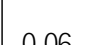 \\
\hline Bilateral & 27 & 46 & 0.00 \\
\hline
\end{tabular}

${ }^{*}$ means $\mathrm{p}<0.05$

MEL: microendoscopic laminotomy. tudinal skin incision of approximately $18 \mathrm{~mm}$ was made beside the $L 4$ spinous process to target the interlaminar space. The approach side (left or right) was the one with dominant leg pain. The fascia incision was slightly longer than the skin incision. Serial tubal dilators of the METRx endoscopic system (Medtronic Sofamor Danek, Memphis, USA) were inserted through the incision. A tubular retractor $16 \mathrm{~mm}$ in diameter was inserted, and secured by the flexible arm mounted to the table side rail. Muscles and soft tissues on the lamina were removed by bipolar cautery and rongeur to enable visualization of the bony edges. The bases of the spinous process, bilateral lamina, and inferior articular processes were drilled by a curved high-speed electric surgical drill (Midas Rex Legend Stylus, Medtronic Sofamor Danek). L4 lamina and both sides of the inferior articular process were resected in the shape of a trumpet to reach the superior attachment of the ligamentum flavum. The cranial part of the L5 lamina was also resected to the inferior attachment of the ligamentum flavum. Until laminotomy was completed, the deep layer of the flavum was preserved to protect the dura and nerve roots. Once all of the attachment sites of the flavum were detached, a lump of flavum floated from the dura and was removed by curved Kerrison rongeur. Medial parts of the superior articular processes of L5 were resected by Kerrison rongeur or osteotome. Finally decompression was achieved to enable identification of both sides of the L5 nerve roots and pulsation of the dura.

\section{Open Fenestration}

Open fenestration has been described in previous literature ${ }^{7)}$. Here we describe briefly the procedure at $L 4 / 5$ (Figure 1). Surgeons approached from the left side of the patients, and made a midline skin incision on the $L 4$ spinous process. Depending on body size, the skin incision usually ranged from $5 \mathrm{~cm}$ to $8 \mathrm{~cm}$. The fascia was incised at the midline and the spinous process and lamina were exposed bilaterally by peeling the paravertebral muscle under the periosteum. The caudal part of the $L 4$ spinous process was resected with the interlamina ligament and supraspinal ligaments. Medial parts of the inferior articular processes of $L 4$ were resected bilaterally in a trumpet shape by osteotome. The ligamentum flavum was removed at the attachment site of the lamina. The medial parts of the superior articular processes of $L 5$ were resected by Kerrison rongeur or osteotome, then the
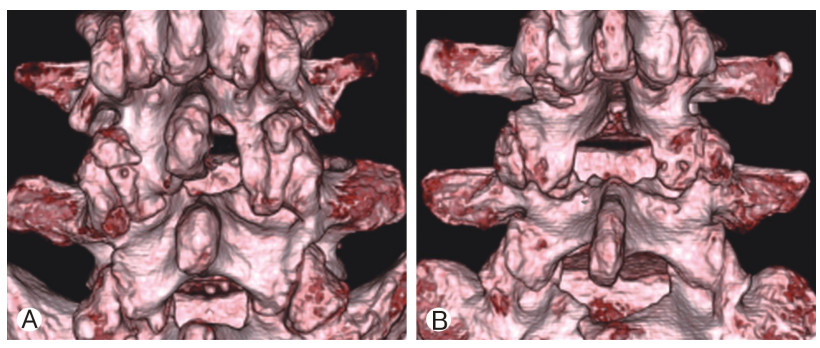

Figure 1. (A): A postoperative 3-dimensional computed tomography of a patient treated with microendoscopic laminectomy from the right side approach. (B): A postoperative 3-dimensional computed tomography of a patient treated with open fenestration. 
dura matter and both sides of the $\mathrm{L} 5$ nerve roots were decompressed.

\section{Outcome Measurements}

\section{1) Surgical Outcomes}

Surgical outcomes recorded were operating time, intraoperative bleeding, postoperative bleeding, surgical complication, and length of postoperative hospital stay. The clinical pathway of single level decompression defines the date of discharge as 7 days to 14 days postoperatively. Patients can choose the discharge date depending on one's condition in consultation with the attending physician.

\section{2) Clinical Outcome}

The Japanese Orthopedic Association Back Pain Evaluation Questionnaire (JOABPEQ) and a visual analog scale (VAS) were routinely used for patients who underwent lumbar surgery in the spine center. Preoperative and 1 year-postoperative scores were used for analysis. The JOABPEQ is a patient-reported health related outcome whose reliability, internal consistency, criteria validity and clinically important difference have been established ${ }^{8-10)}$. The JOABPEQ consists of 25 items divided into 5 domains: low back pain, lumbar function, walking ability, social function, and mental health. The questionnaire is set up so that scores in each domain range from 0 to 100 , with higher scores indicating a better condition. The clinically important difference for each domain is 20 points ${ }^{11,12)}$.

\section{3) Visual Analog Scale}

A VAS was used to measure perceived pain in three parts of the body: The low back, the buttocks and lower limbs, and numbness in the buttocks and lower limbs. Zero means no pain and 100 means the most intense pain imaginable.

\section{4) Radiographic Outcome}

Preoperative and postoperative radiography and CT scans were routinely performed for patients who underwent spine surgery in the spine center. Radiographic parameters of interest at the surgical level included; (1) range of motion (ROM) of the intervertebral disc in flexion-extension radiograph, (2) disc height, (3) postoperative vertebral slippage $(>3 \mathrm{~mm})$. Postoperative radiographs were taken one year after surgery.

CT parameters of interest ${ }^{13)}$ (Figure 2) at the surgical level included; (1) resection width of the inferior articular process, (2) resection angle of the inferior articular process, (3) resection width of the superior articular process. Width of the articular processes was measured at the cranial margin of the pedicle using multi-planar reconstruction images in a digital viewer (SYNAPSE VINCENT, FUJIFILM).

\section{5) Blood Test Outcome}

Blood test outcomes included hemoglobin level $(\mathrm{Hb})$, total protein (TP), albumin (Alb), creatine kinase (CK), and C-reactive protein (CRP). Blood tests were performed three days after surgery based on the clinical pathway.

\section{6) Statistical Analysis}

A Mann-Whitney $U$ test was used to compare the parameters without normal distribution, such as preoperative and postoperative JOABPEQ. Other parameters and changes in JOABPEO scores were compared by using a student's $t$ test ${ }^{10,11)}$. The Fisher's exact test was used to compare the categorical data. A p value of less than 0.05 (two-tailed) was considered statistically significant. When the 95\% confidence interval $(\mathrm{Cl})$ for between-group differences in JOABPEQ score change was within the clinically important difference ( -20 to 20$)$, the groups were considered as clinically equivalent. The statistical analysis was performed with SPSS Statistics (version 20; IBM, Armonk, NY, USA).

\section{RESULTS}

\section{Patient Demographic}

Thirty patients in the MEL group and 46 patients in the fenestration group met the criteria. Patients' demographic data are shown in Table 1. There was no significant difference in age, sex, or BMl between the groups. In the MEL group, there were 22 males and 8 females, with a mean age of $67 \pm 7$ years old.

In the fenestration group, there were 43 males and 13 females, with a mean age of $70 \pm 10$ years old.

\section{Surgical Outcomes}

Surgical outcomes are shown in Table 2. Operating time was
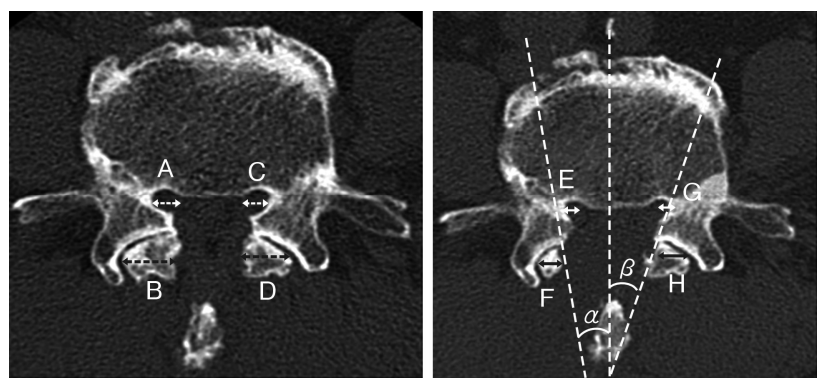

Figure 2. An example of CT parameters in a case of MEL from the right-side approach. A : Preoperative width of the superior articular process on the approach side. B: Preoperative width of the inferior articular process on the approach side. $\mathrm{C}$ : Preoperative width of the superior articular process on the contralateral side. D: Preoperative width of the inferior articular process on the contralateral side. $\mathrm{E}$ : Postoperative width of the superior articular process on the approach side. F: Postoperative width of the inferior articular process on the approach side. G: Postoperative width of the superior articular process on the contralateral side. $\mathrm{H}$ : Postoperative width of the inferior articular process on the contralateral side. $\boldsymbol{\alpha}$ : The resection angle of the inferior articular process on the approach side. $\beta$ : The resection angle of the inferior articular process on the contralateral side. 
significantly longer in the MEL group (122 $\mathrm{min}$ ) than in the fenestration group (39 min; $p<0.001$ ). Intraoperative bleeding was similar in the MEL group (44 $\mathrm{ml}$ ) than in the fenestration group (35 $\mathrm{ml} ; \mathrm{p}=0.5$ ). There was significantly less postoperative bleeding in the MEL group $(87 \mathrm{ml})$ than in the fenestration group (190 $\mathrm{ml} ; \mathrm{p}<0.001)$. Postoperative hospital stays were significantly shorter in the MEL group (9 days) than in the fenestration group (13 days; $\mathrm{p}=0.001$ ).

There were one case in the MEL group and two cases of dural tear in the fenestration group $(p=1.0)$. There were no case of postoperative hematoma or iatrogenic pars fracture in either group.

\section{Clinical Outcomes}

Clinical outcomes are shown in Table 3.

\section{JOABPEQ}

There was no significant differences in score changes of either domains of JOABPEQ between MEL and fenestration.

Table 2. Comparison of surgical outcomes between the MEL group and the fenestration group

\begin{tabular}{l|c|c|l}
\hline \hline & MEL & Fenestration & p-value \\
\hline Operative time (min) & $122 \pm 39$ & $39 \pm 11$ & $<0.001^{*}$ \\
Intraoperative blood loss (ml) & $44 \pm 57$ & $35 \pm 64$ & 0.5 \\
Postoperative blood loss (ml) & $87 \pm 82$ & $190 \pm 73$ & $<0.001^{*}$ \\
Hospital stay after surgery (days) & $8.6 \pm 2.0$ & $12.5 \pm 6.3$ & $0.001^{*}$ \\
Dural tear (case) & 1 & 2 & 1.0 \\
Pars fracture (case) & 0 & 0 & 1.0 \\
Epidural hematoma (case) & 0 & 0 & 1.0 \\
Postoperative slippage (case) & 0 & 0 & 1.0 \\
\hline
\end{tabular}

*means $\mathrm{p}<0.05$.

MEL: microendoscopic laminotomy.
The difference in score change for low back pain was 2 point better in MEL than fenestration ( $95 \% \mathrm{Cl} ;-15$ to 19). The difference in score change for lumbar function was 1 point better in MEL than fenestration $(95 \% \mathrm{Cl} ;-10$ to 13$)$. The difference in score change for walking ability was 2 point better in fenestration than MEL (95\% Cl; -17 to 13$)$. The difference in score change for social function was 1 point better in MEL than fenestration $(95 \% \mathrm{Cl} ;-10$ to 13$)$. The difference in score change for mental health was 2 point better in fenestration than MEL $(95 \% \mathrm{Cl}$; -10 to 6$)$. Thus the $95 \% \mathrm{Cls}$ of the between-group differences in score change was within \pm 20 in all domains (Figure 3 ).

\section{Visual Analogue Scale}

There was no significant score change between-group differences except low back pain. The difference in change for VAS of pain in low back was 20 point better in MEL than fenestration (95\% Cl; -61 to -32$)$. The difference in change for VAS of pain in buttocks and lower limb was 10 point better in MEL than fenestration (95\% Cl; -27 to 5). The difference in change for VAS of numbness in buttocks and lower limb was 7 point better in MEL than fenestration ( $95 \% \mathrm{Cl} ;-18$ to 11$)$.

\section{Radiographic Outcomes}

\section{Radiographic outcomes are shown in Table 4.}

\section{1) Disc Height and ROM at the Surgical Site}

In MEL, at the surgical site preoperative and postoperative disc height were $10.7 \mathrm{~mm}$ and $10.5 \mathrm{~mm}$, respectively. In fenestration, preoperative and postoperative disc height were $9.6 \mathrm{~mm}$ and $9.5 \mathrm{~mm}$, respectively. There was no significant difference in change of disc height between the two groups $(-0.2 \mathrm{~mm}$ vs $-0.1 \mathrm{~mm}, \mathrm{p}=0.3)$.

In MEL, preoperative and postoperative ROM at the surgical

Table 3. Comparison of clinical outcomes between the MEL group and the fenestration group

\begin{tabular}{|c|c|c|c|c|c|c|c|c|c|c|c|}
\hline & & \multicolumn{3}{|c|}{ Pre } & \multicolumn{3}{|c|}{ Post } & \multicolumn{3}{|c|}{ Change } & \multirow{2}{*}{$\begin{array}{l}\text { Difference in change } \\
\text { between groups } \\
(95 \% \mathrm{Cl})\end{array}$} \\
\hline & & MEL & $\begin{array}{l}\text { Fenest- } \\
\text { ration }\end{array}$ & $p$-value & MEL & \begin{tabular}{|l|} 
Fenest- \\
ration
\end{tabular} & $p$-value & MEL & \begin{tabular}{|l} 
Fenest- \\
ration
\end{tabular} & $p$-value & \\
\hline \multirow{5}{*}{$\begin{array}{l}\text { JOA } \\
\text { BPEQ }\end{array}$} & Low back pain & $47 \pm 35$ & $52 \pm 32$ & 0.5 & $78 \pm 31$ & $81 \pm 30$ & 0.6 & $31 \pm 39$ & $29 \pm 35$ & 0.8 & $2(-15$ to 19$)$ \\
\hline & Lumbar function & $64 \pm 28$ & $70 \pm 28$ & 0.4 & $78 \pm 22$ & $82 \pm 19$ & 0.5 & $14 \pm 20$ & $13 \pm 26$ & 0.8 & $1(-10$ to 13$)$ \\
\hline & Walking ability & $39 \pm 29$ & $34 \pm 25$ & 0.4 & $78 \pm 28$ & $75 \pm 30$ & 0.6 & $39 \pm 27$ & $41 \pm 36$ & 0.8 & $-2(-17$ to 13$)$ \\
\hline & Social function & $49 \pm 26$ & $45 \pm 20$ & 0.5 & $72 \pm 19$ & $67 \pm 24$ & 0.3 & $23 \pm 27$ & $22 \pm 24$ & 0.8 & $1(-10$ to 13$)$ \\
\hline & Mental health & $54 \pm 15$ & $50 \pm 14$ & 0.3 & $61 \pm 18$ & $59 \pm 17$ & 0.7 & $7 \pm 18$ & $9 \pm 17$ & 0.6 & $-2(-10$ to 6$)$ \\
\hline \multirow{3}{*}{ VAS } & Pain in low back & $59 \pm 28$ & $49 \pm 30$ & 0.2 & $16 \pm 19$ & $27 \pm 28$ & 0.09 & $-43 \pm 30$ & $-23 \pm 28$ & $0.005^{*}$ & -20 (-61 to -32$)$ \\
\hline & $\begin{array}{l}\text { Pain in buttocks and } \\
\text { lower limb }\end{array}$ & $64 \pm 27$ & $58 \pm 24$ & 0.4 & $20 \pm 22$ & $25 \pm 29$ & 0.4 & $-44 \pm 32$ & $-34 \pm 36$ & 0.2 & $-10(-27$ to 5$)$ \\
\hline & $\begin{array}{l}\text { Numbness in buttocks } \\
\text { and lower limb }\end{array}$ & $59 \pm 27$ & $61 \pm 25$ & 0.7 & $20 \pm 24$ & $30 \pm 32$ & 0.2 & $-39 \pm 33$ & $-32 \pm 34$ & 0.4 & $-7(-18$ to 11$)$ \\
\hline
\end{tabular}


site were $2.2^{\circ}$, and $2.8^{\circ}$, respectively. In fenestration, preoperative and postoperative ROM were $1.2^{\circ}$ and $1.9^{\circ}$, respectively. There was no significant between-group difference in change of ROM $\left(0.6^{\circ}\right.$ vs $\left.0.7^{\circ}, p=0.9\right)$. There was no postoperative slippage in either group.

\section{Resection Width of Facet on CT}

\section{1) Approach Side}

On the approach side, the resection width of the inferior articular process was significantly larger in the MEL group $(3.5 \mathrm{~mm})$ than in the fenestration group $(2.6 \mathrm{~mm} ; \mathrm{p}=0.03)$. The resection angle of the inferior articular process was significantly larger in the MEL group $\left(-0.4^{\circ}\right)$ than in the fenestration group $\left(11.7^{\circ}\right.$; $\mathrm{p}<0.001)$. There was no significant difference in resection width of the superior articular process between the MEL group (2.3 $\mathrm{mm})$ and the fenestration group $(3.0 \mathrm{~mm} ; \mathrm{p}=0.06)$.

\section{2) Contralateral Side}

On the contralateral side, there was no significant difference in resection width of the inferior articular process between the MEL group $(2.9 \mathrm{~mm})$ and the fenestration group $(2.5 \mathrm{~mm} ; \mathrm{p}=1.0)$. The resection angle of the inferior articular process was significantly larger in the MEL group $\left(27.5^{\circ}\right)$ than in the fenestration group $\left(15.2^{\circ} ; p<0.001\right)$. The resection width of the superior articular process was significantly smaller in the MEL group $(2.4 \mathrm{~mm})$ than in the fenestration group $(3.1 \mathrm{~mm} ; \mathrm{p}=0.03)$.

\section{3) Blood Test Outcome}

Blood test outcomes are shown in Table 5. The increase in CRP was significantly higher in the fenestration group $(2.9 \mathrm{mg} /$ $\mathrm{dL})$ than in the MEL group $(1.7 \mathrm{mg} / \mathrm{dL} ; \mathrm{p}<0.001)$. There were no significant between-group differences in decrease in $\mathrm{Hb}$, loss of $\mathrm{TP}$, loss of Alb, or increase of CK.

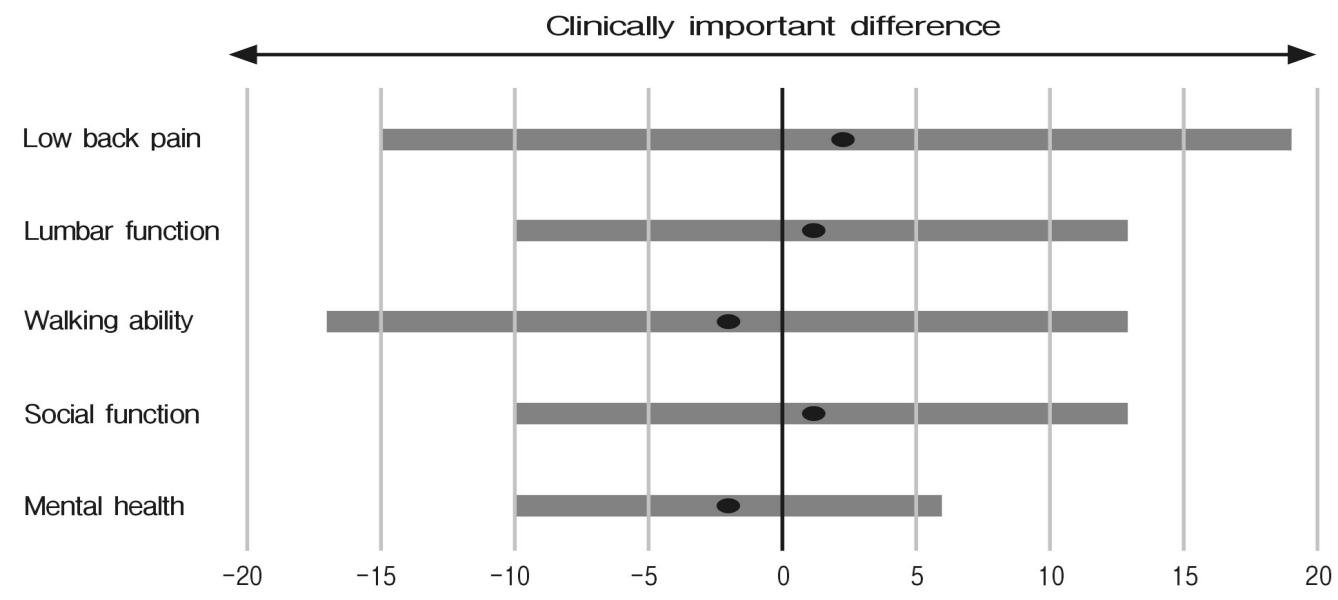

Figure 3. Clinical equivalence between MEL and open fenestration in each domain of JOABPEQ. Black dot shows mean difference of score change from preoperatively to postoperatively between MEL and Fenestration. Blue bar shows the $95 \%$ confidence interval of the difference. Each bar falls within clinically important difference of JOABPEQ $( \pm 20)$.

Table 4. Comparison of radiographic outcomes between the MEL group and the fenestration group

\begin{tabular}{|c|c|c|c|c|c|c|c|c|c|c|c|}
\hline & & \multicolumn{3}{|c|}{ Pre } & \multicolumn{3}{|c|}{ Post } & \multicolumn{3}{|c|}{ Change } \\
\hline & & & MEL & $\begin{array}{l}\text { Fenest- } \\
\text { ration }\end{array}$ & $p$-value & MEL & $\begin{array}{c}\text { Fenest- } \\
\text { ration }\end{array}$ & $\mathrm{p}$-value & MEL & $\begin{array}{l}\text { Fenest- } \\
\text { ration }\end{array}$ & p-value \\
\hline \multirow{2}{*}{\multicolumn{2}{|c|}{$\begin{array}{l}\text { Plain } \\
\text { radiography }\end{array}$}} & Disc height $(\mathrm{mm})$ & $10.7 \pm 2.4$ & $9.6 \pm 2.9$ & 0.1 & $10.5 \pm 2.6$ & $9.5 \pm 3.1$ & 0.5 & $-0.2 \pm 0.8$ & $0.1 \pm 0.9$ & 0.3 \\
\hline & & Range of motion (degree) & $2.2 \pm 2.6$ & $1.2 \pm 3.6$ & 0.2 & $2.8 \pm 2.6$ & $1.9 \pm 2.5$ & 0.2 & $0.6 \pm 3.8$ & $0.7 \pm 4.3$ & 0.9 \\
\hline \multirow{4}{*}{ CT } & Approach & $\begin{array}{l}\text { Width of the inferior } \\
\text { articular process }(\mathrm{mm})\end{array}$ & $10.2 \pm 2.4$ & $9.3 \pm 1.9$ & 0.1 & $6.6 \pm 2.6$ & $6.7 \pm 1.7$ & 1.0 & $3.5 \pm 1.8$ & $2.6 \pm 1.7$ & $0.03^{*}$ \\
\hline & side & $\begin{array}{l}\text { Width of the superior } \\
\text { articular process }(\mathrm{mm})\end{array}$ & $6.0 \pm 3.1$ & $5.4 \pm 1.5$ & 0.2 & $3.7 \pm 3.0$ & $2.2 \pm 1.5$ & $0.01^{*}$ & $2.3 \pm 1.4$ & $3.0 \pm 1.7$ & 0.06 \\
\hline & \multirow{2}{*}{$\begin{array}{l}\text { Contralat } \\
\text { eral side }\end{array}$} & $\begin{array}{l}\text { Width of the inferior } \\
\text { articular process }(\mathrm{mm})\end{array}$ & $8.9 \pm 2.3$ & $9.3 \pm 2.1$ & 0.2 & $6.3 \pm 2.8$ & $6.7 \pm 2.1$ & 0.5 & $2.9 \pm 2.6$ & $2.5 \pm 1.4$ & 1.0 \\
\hline & & $\begin{array}{l}\text { Width of the superior } \\
\text { articular process }(\mathrm{mm})\end{array}$ & $6.0 \pm 3.3$ & $5.3 \pm 1.5$ & 0.2 & $3.6 \pm 3.6$ & $2.3 \pm 1.5$ & $0.003^{*}$ & $2.4 \pm 2.6$ & $3.1 \pm 1.2$ & $0.03^{*}$ \\
\hline
\end{tabular}

*Means $\mathrm{p}<0.05$. CT: computed tomography, MEL: microendoscopic laminotomy. 
Table 5. Comparison of blood test outcomes between the MEL group and the fenestration group

\begin{tabular}{l|c|c|c|c|c|c|c|c|c}
\hline & \multicolumn{3}{|c|}{ Pre } & \multicolumn{3}{c|}{ Post } & \multicolumn{3}{c}{ Change } \\
\hline \hline & MEL & Fenestration & p-value & MEL & Fenestration & $p$-value & MEL & Fenestration & p-value \\
\hline Hemoglobin & $14.4 \pm 1.2$ & $14.1 \pm 1.3$ & 0.4 & $13.1 \pm 1.3$ & $12.9 \pm 1.5$ & 0.5 & $-1.3 \pm 0.9$ & $-1.3 \pm 0.8$ & 1.0 \\
Total protein (g/dL) & $7.2 \pm 0.4$ & $7.1 \pm 0.5$ & 0.3 & $5.9 \pm 0.4$ & $6.0 \pm 0.4$ & 0.07 & $-1.3 \pm 0.4$ & $-1.1 \pm 0.4$ & 0.06 \\
Albumin (g/dL) & $4.4 \pm 0.3$ & $4.3 \pm 0.3$ & 0.2 & $3.5 \pm 0.3$ & $3.6 \pm 0.4$ & 0.4 & $-0.9 \pm 0.3$ & $-0.8 \pm 0.3$ & 0.3 \\
Creatine kinase (u/L) & $126 \pm 64$ & $132 \pm 80$ & 0.7 & $176 \pm 85$ & $183 \pm 99$ & 0.7 & $50 \pm 88$ & $51 \pm 92$ & 1.0 \\
C-reactive protein $(\mathrm{mg} / \mathrm{dL})$ & $0.3 \pm 1.1$ & $0.1 \pm 0.3$ & 0.5 & $2.0 \pm 1.6$ & $3.2 \pm 2.3$ & $0.02^{*}$ & $1.7 \pm 2.2$ & $2.9 \pm 1.9$ & $0.009^{*}$ \\
\hline
\end{tabular}

${ }^{*}$ Means $\mathrm{p}<0.05$.

CT: Computed tomography, MEL: microendoscopic laminotomy.

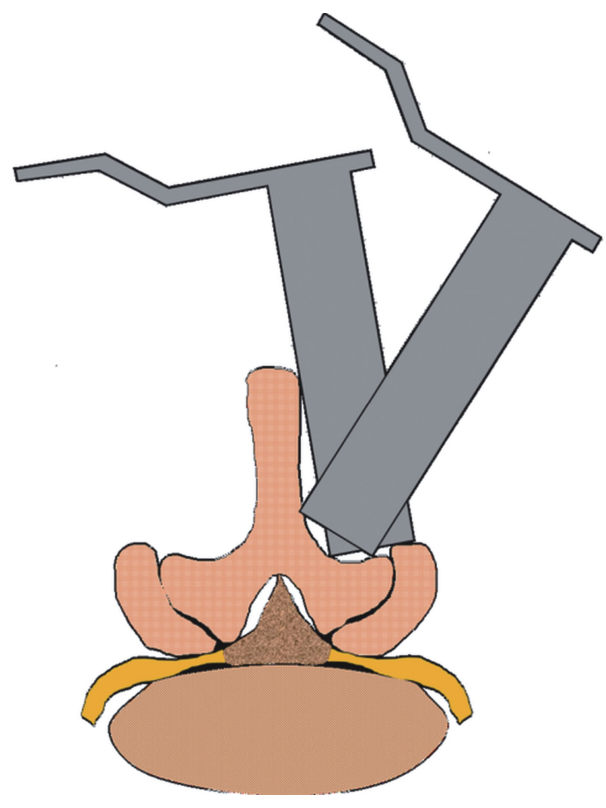

Figure 4. Handling of the tubular retractor. When surgeons see the facet on the approach side, the tubular retractor collides with the spinous process. To see the contralateral side, surgeons can incline the retractor.

\section{DISCUSSION}

Although there have been some previous studies of MEL, most of these studies have been limited to case series, ${ }^{3,6)}$ and only one study has included open procedures as the control group ${ }^{5}$. Our study was the first to compare the patient-reported clinical outcomes of MEL and fenestration for lumbar spinal stenosis. The 95\% Cls of the between-group differences in score change were within clinical important difference ( \pm 20 point) in all domains of JOABPEO. The improvement of VAS in low back pain was better in MEL than fenestration. The clinical important difference of VAS was reported to be 15 to 25 point $^{14,15)}$. These data demonstrated that MEL had as equivalent clinical effectiveness as open fenestration did.

The MEL group had significantly shorter hospital stays (4 days shorter) and a lower increase in CRP $(1.2 \mathrm{mg} / \mathrm{dL}$ lower) than the fenestration group. However, the MEL had limitations in regard to operating time. There was no significant difference in other blood test parameters. Because surgical invasiveness of fenestration at single level is not very high, it may be difficult for MEL to gain large advantages in invasiveness over fenestration. In more invasive surgery at multilevel, MEL may have more advantage over open fenestration. However, shortening of operation time may be necessary for MEL to maintain the advantages. Longer operation time is a concern about invasiveness of MEL. There are some possible reasons for longer operation time. Because endoscopic surgery is relatively new technique, we have less opportunity to learn endoscopic techniques from the senior physicians. We need to develop our own techniques by trial and error. Thus the learning curve of endoscopic surgery is likely to be shallower than that of conventional open procedures. Moreover, surgical equipment like rongeur in endoscopic surgery is smaller than that of open surgery and resection volume of bone and flavum in one action is limited. In resection of the bone, we use osteotome in open fenestration but use high speed drill in MEL. It takes more time to grind bone by drill than to resect a lump of bone by osteotome. Endoscope is sometimes soiled by bone dust and needs clean out. These factors are associated with longer operation time of MEL. In recent cases, we use osteotome more frequently in MEL and succeed to reduce surgical time.

The CT data demonstrated that the resection angle of the inferior articular process on the approach side was significantly smaller in the MEL group than in the fenestration group. The tubular retractor collides with the spinous process, making it difficult to undercut the facet in a trumpet shape (Figure 4) ${ }^{5}$. As a result, the resection length of the inferior articular process on the approach side was larger in MEL than in fenestration. However, this phenomenon can occur also in open procedures when the spinous process is preserved like in Love method (nucleotomy). The difference of resection length of the inferior articular process between MEL and fenestration was $1 \mathrm{~mm}$. No cases showed postoperative slippage. These results demonstrated that resection of the inferior articular process on the approach side in MEL had little impact on spinal stability. Although the resection of the superior articular process in MEL was smaller than that in fenestration, improvement of leg pain was equivalent to fenestration. Flavectomy around the superior articular process achie- 
ved neural decompression.

There have been some previously published studies examining microendoscopic spinal surgery ${ }^{3,5,16)}$. Minamide et al. reported a case series of 304 patients who were treated with microendoscopic laminotomy using the METRx system ${ }^{3}$. They reported that $69 \%$ of the patients were good or excellent and $20 \%$ of the patients were fair based on the surgeon-reported conventional Japanese Orthopedic Association scoring system. Pao et al. reported a case series of 53 patients treated with MEL. The Oswestry Disability Index scores of the patients improved from 64 to 17. lkuta et al. compared the clinical outcomes of 44 patients treated with MEL and 29 patients treated with microscopic laminotomy $y^{5}$. Although the improvement rate according to the conventional JOA scoring system was 70\% in both groups, the complication rate for MEL was $25 \%$ and that was significantly higher than that for open laminotomy. The reason for the high complication rate may be that their study included early cases patients after introduction of microendoscopic surgery. The surgeons in our study were certified by the endoscopic surgical skill qualification system and started MEL after an experience of a certain number of MED cases.

There were some limitations in our study, specifically that it was a retrospective design and patients were not randomized.

In conclusion, MEL is as effective a treatment option as open fenestration for lumbar spinal stenosis. MEL is less invasive, having a smaller skin incision, shorter hospital stay, and lower increase in CRP. On the other hand, the longer operating time and learning curve of MEL are issues to consider when determining its suitability for more complicated spinal disorders that need multiple level decompression or fusion.

\section{CONFLICT OF INTEREST}

No potential conflict of interest relevant to this article.

\section{REFERENCES}

1. Perez-Cruet MJ, Foley KT, Isaacs RE, et al.: Microendoscopic lumbar discectomy: Technical note. Neurosurgery 51(5 Suppl): S129-136, 2002

2. Ishii K: The current status of endoscopic spinal surgery 2016. (in Japanese). J Jpn Orthop Assoc 92(1):56-62, 2018

3. Minamide A, Yoshida M, Simpson AK, et al.: Minimally invasive spinal decompression for degenerative lumbar spondylolisthesis and stenosis maintains stability and may avoid the need for fusion. Bone Joint J 100-B(4):499-506, 2018

4. Yoon JW, Wang MY: The evolution of minimally invasive spine surgery. J Neurosurg Spine 30(2):149-158, 2019

5. Ikuta K, Arima J, Tanaka T, et al.: Short-term results of microendoscopic posterior decompression for lumbar spinal stenosis.
Technical note. J Neurosurg Spine 2(5):624-633, 2005

6. Minamide A, Yoshida M, Yamada H, et al.: Endoscope-assisted spinal decompression surgery for lumbar spinal stenosis. J Neurosurg Spine 19(6):664-671, 2013

7. Kanamori M, Matsui H, Hirano N, Kawaguchi Y, Kitamoto R, Tsuji H: Trumpet laminectomy for lumbar degenerative spinal stenosis. J Spinal Disord 6(3):232-237, 1993

8. Fukui M, Chiba K, Kawakami M, et al.: Japanese Orthopaedic Association Back Pain Evaluation Questionnaire. Part 3. Validity study and establishment of the measurement scale: Subcommittee on Low Back Pain and Cervical Myelopathy Evaluation of the Clinical Outcome Committee of the Japanese Orthopaedic Association, Japan. J Orthop Sci 13(3):173-179, 2008

9. Fukui M, Chiba K, Kawakami M, et al.: Japanese Orthopaedic Association Back Pain Evaluation Questionnaire. Part 2. Verification of its reliability: The Subcommittee on Low Back Pain and Cervical Myelopathy Evaluation of the Clinical Outcome Committee of the Japanese Orthopaedic Association. J Orthop Sci 12 (6):526-532, 2007

10. Fukui M, Chiba K, Kawakami M, et al.: JOA Back Pain Evaluation Questionnaire (JOABPEQ)/JOA Cervical Myelopathy Evaluation Questionnaire (JOACMEQ). The report on the development of revised versions. April 16, 2007. The Subcommittee of the Clinical Outcome Committee of the Japanese Orthopaedic Association on Low Back Pain and Cervical Myelopathy Evaluation. J Orthop Sci 14(3):348-365, 2009

11. Japanese Orthopaedic Association, The committee of diagnosis and evaluation criteria of the Japanese Society for Spine Surgery and Related Research. The user manual of JOABPEQ and JOACMEQ (in Japanese). Tokyo: Nankodo; 2012.

12. Fujimori T, Miwa T, Oda T: Responsiveness of the Japanese Orthopaedic Association Back Pain Evaluation Questionnaire in lumbar surgery and its threshold for indicating clinically important differences. Spine J 19(1):95-103, 2019

13. Dohzono S, Matsumura A, Terai $H$, Toyoda $H$, Suzuki A, Nakamura $\mathrm{H}$ : Radiographic evaluation of postoperative bone regrowth after microscopic bilateral decompression via a unilateral approach for degenerative lumbar spondylolisthesis. J Neurosurg Spine 18(5):472-478, 2013

14. Beck EC, Nwachukwu BU, Kunze KN, Chahla J, Nho SJ: How Can We Define Clinically Important Improvement in Pain Scores After Hip Arthroscopy for Femoroacetabular Impingement Syndrome? Minimum 2-Year Follow-up Study. Am J Sports Med 47 (13):3133-3140, 2019

15. Martin RL, Kivlan BR, Christoforetti JJ, et al.: Minimal Clinically Important Difference and Substantial Clinical Benefit Values for a Pain Visual Analog Scale After Hip Arthroscopy. Arthroscopy 35(7):2064-2069, 2019

16. Pao JL, Chen WC, Chen PQ: Clinical outcomes of microendoscopic decompressive laminotomy for degenerative lumbar spinal stenosis. Eur Spine J 18(5):672-678, 2009 